\title{
Relationship Between Leukocyte, ESR, and CRP Infection Markers with Changes in Wound Wagner 2 and 3 Diabetic Foot Ulcers \\ Patrianef Darwis ${ }^{a *}$, Jimmy Candra Putra ${ }^{b}$, Dedy Pratama ${ }^{a}$, Aria Kekalih ${ }^{c}$
}

Introduction: In 2012, the annual incidence rate of diabetic foot ulcers and gangrene are estimated to be around $2-5 \%$ of the general population. About $15 \%$ of patients with diabetic foot can have an amputation in the lower limb. Diabetic foot is a health problem that is very difficult to cure. This is further exacerbated by severe conditions of infection and disrupt the process of tissue regeneration, so amputation must be done to prevent the spread of infection. Infection that is not well controlled can inhibit all phases of wound healing. This study aimed to know the relationship between laboratory profile and wound healing in diabetic foot ulcer patients.

Method: This was a cross-sectional study with research subjects who were diabetic foot ulcer patients who went to the emergency room and the Cipto Mangunkusumo National Hospital polyclinic during August-December 2019. Statistical analysis was performed to see the correlation between changes in infection marker values and changes in the wound area.

Results: During the period August 2019 to December 2019, 30 subjects met the inclusion and exclusion criteria. There were 14 subjects (46.77\%) male and 16 subjects $(53.3 \%)$ female. From the diagnosis, there were 20 subjects $(66.3 \%)$ with ulcer pedis and ten subjects (33.3\%) gangrene pedis. From this study, the average and standard deviation of changes in $A B I$ values were $0.9080 \pm 0.100$, changes in the number of leukocytes amounted to $4899.87 \pm 4512.048$, changes in ESR values was $1.8333 \pm 1.147$, changes in CRP values was $2.6500 \pm 1.702$, changes in wound area was $10.2727 \pm 6.512$, and albumin was $2.9487 \pm$ 0.392 . From the correlative analysis, it was found between changes in the number of leukocytes with changes in wound area $(p=0.058, r=0.350)$, changes in ESR values with changes in wound area $(p=0.034, r=0.388)$, and changes in CRP values with changes in wound area $(p=0.008, r=0.477)$.

Conclusion: There was a significant relationship between changes in ESR values and CRP values with moderate correlation strength changes in the wound area. There was no significant relationship between changes in the number of leukocytes with changes in the wound area.

Keywords: wound area, leukocytes, CRP, ESR, diabetic foot ulcer, leukocytes. https://doi.org/10.36864/jinasvs.2020.2.007

*Correspondence: patrianef@gmail.com

aM.D., Vascular and Endovascular Division, Department of Surgery, Cipto Mangunkusumo Hospital - Faculty of Medicine, Universitas Indonesia, Jakarta, Indonesia

${ }^{b}$ M.D., Department of Surgery, Dr. Soeroto Hospital, East Java, Indonesia

cM.D., M.T.I., Ph.D., Department of Community Medicine, Faculty of Medicine Universitas Indonesia, Jakarta, Indonesia

\section{INTRODUCTION}

Cipto Mangunkusumo Hospital, as a national referral hospital in Indonesia, receives many diabetic foot ulcers through the Emergency Department (ED) and the clinic. Almost all cases are referrals from hospitals originating from all over Indonesia, although most cases remain from Jakarta and surrounding areas.

Based on the 2012 report, the global prevalence of diabetes mellitus (DM) worldwide is expected to increase gradually to exceed 5.9 million
Copyright (C) 2020, The Indonesian Society for Vascular and Endovascular Surgery

JINASVS 2020;1(2):26-29 in 2030.1,2 Data from the World Health Organization (WHO) stated that the number of people with DM in Indonesia had increased around 2-3 times from 8.4 million in 2000 to 21.3 million in 2030 . Also, data from the International Diabetes Federation (IDF) in 2014 stated that Indonesia had the 5th most significant number of DM sufferers worldwide, with a total of about 9.1 million. Data from the Basic Health Research in Indonesia in 2018 states that the prevalence of DM for ages above 15 years was $6.9 \% .^{3}$ The same data also stated around $40-70 \%$ of 
amputation cases in the lower extremities due to nontrauma caused by diabetic foot. ${ }^{1,4}$

Diabetic foot ulcer (DFU) in terminology is all lesions that occur in the foot due to diabetes or its complications. The principle of management of diabetic foot ulcers is the closure of the wound as soon as possible. Management of DFU is carried out comprehensively, including debridement, reducing pressure load, infection control, and foot care. ${ }^{5}$ Diabetes foot is one of the severe complications because it is still a complicated problem and has not been managed optimally. The causes include the fact that there are still very few people who work individually on the feet of diabetes and there is no education on the management of diabetes specifically, so that diabetes often ends in disability and death. ${ }^{6,7}$

This study aimed to see whether there was a relationship between a decrease in the percentage of infection markers and a decrease in the percentage of diabetic foot injuries. Markers of infection to be examined were the number of leukocytes, C-reactive protein (CRP), and erythrocyte sedimentation rate (ESR) because infection is an important factor in wound healing. This study used leukocyte, ESR, and CRP infection markers because they are easy to do in almost all hospitals. This research is expected to help determine the prediction of the length of treatment and the possibility of amputation in diabetic foot patients.

\section{METHOD}

The research design used was a crosssectional design. Diabetic foot ulcer patients included in this study had undergone a series of examinations, which were measurement of wound area, examination of infection markers (leukocyte counts, C-reactive protein, and erythrocyte sedimentation rate), anklebrachial index (ABI), and foot X-rays. The study was conducted at the Emergency Department (ED) and the Cipto Mangunkusumo National Hospital Polyclinic. The research period was done for four months, from August 2019 to December 2019.

Criteria for inclusion of patients in this study were diabetic foot wound patients undergoing treatment in the ER or Cipto Mangunkusumo National Hospital, diabetic foot injury patients of degrees 2-3 according to the Wagner-Meggit classification, with notes for grade 3 undergoing bone chipping during debridement, patients who require wound care and debridement, and is willing to sign informed consent. Patients with sepsis, severe PAD $(A B I<0.40)$, immunocompromise, source of infection elsewhere, CKD with routine hemodialysis, and patients requiring redebridement were excluded from the study.

Venous blood sampling for laboratory tests will be performed on the right or left arm concerning aseptic and antiseptic actions. Blood will be taken as much as $3 \mathrm{ml}$ and put in a sterile tube containing anticoagulants prepared and then sent to a laboratory to check the number of leukocytes, CRP levels, and ESR values.

Subjects will undergo treatment in the form of antibiotics, regular dressing, or debridement according to existing guidelines. After the third day of debridement, the patient will again undergo laboratory tests to check the number of leukocytes, CRP levels and ESR values. The area of the wound will be measured using the Lesion Meter ${ }^{\mathrm{TM}}$ application, which can be downloaded on a smartphone. After one month of treatment, the subject will again undergo a physical examination to determine the degree of depth of the wound and measure the extent of the wound.

\section{RESULTS}

This study obtained a total of 30 patients with diabetic foot injuries without severe PAD at Cipto Mangunkusumo National Hospital. There were 14 patients $(46.77 \%)$ male and 16 patients $(53.3 \%)$ female. The diagnosis obtained as many as 20 patients $(66.3 \%)$ with ulcer pedis and as many as ten patients $(33.3 \%)$ with gangrene pedis.

Table 1. Laboratory profile of patients before and after three days of treatment.

\begin{tabular}{lll}
\hline Variable & Median & (Min-max) \\
\hline ABI & 0.8 & +0.07 \\
$\Delta$ Leukocyte & 3245 & $(100-17880)$ \\
$\Delta$ ESR & 2 & $(0-4)$ \\
$\Delta$ CRP & 2.2 & $(0.0-7.1)$ \\
$\Delta$ Wound size & 9.5 & $(2.07-26.77)$ \\
Albumin & 2.9 & +0.3 \\
\hline
\end{tabular}

This study looked for an association between changes in the value of infection markers with changes in wound area in diabetic foot injury patients. From the analysis of this study, there was no statistically significant relationship between changes in the number of leukocytes with changes in the area of diabetes foot injury $(p=0.058)$ but showed a positive correlation and had a moderate correlation $(r=0.350)$. There was a significant relationship between changes in CRP levels and changes in the area of diabetic foot injury $(p=0.008)$ and a moderate correlation $(r=0.477)$. There was a significant relationship between changes in the value of ESRs with the area of diabetes foot injury $(p=0.034)$ and had a moderate correlation $(r=0.388)$.

Tabel 2. Correlation between variables with percentage changes in wound size.

\begin{tabular}{lcc}
\hline Variable & $\begin{array}{c}\text { Percentage changes in wound size } \\
\text { Power }(r)\end{array}$ \\
\hline Leukocyte & 0.058 & 0.350 \\
ESR & 0.034 & 0.388 \\
CRP & 0.008 & 0.477 \\
\hline
\end{tabular}

*Spearman correlation method

From the table above, it is shown that the percentage of wound change was directly proportional to the change in ESR and CRP so that the greater the difference in ESR and CRP compared to the initial condition, the greater the wound repair. Changes in leukocytes showed a positive correlation but not statistically significant.

\section{DISCUSSION}

This study used markers of leukocyte infection, ESR, and CRP because this examination is easy to do, the cost is not too high, and can be done in almost all hospitals.

Research conducted by Coeprer et al., eight also assessed diabetic foot areas within four weeks after treatment, obtaining a significant result between healing with the laboratory test results marking infection. In the study of Coeprer, et al. ${ }^{8}$ assessed the size of the diabetic foot within four weeks after treatment would predict wound healing in the next time. Patients were called responders or good if 
wound healing was $>50 \%$, and if wound healing $<50 \%$ (non-responder) was considered wound healing was not good. It was also stated that the probability of wound healing after 12 weeks of treatment was $52.3 \%$ (responder) and $18.4 \%$ (nonresponder), the probability of wound healing after 16 weeks of wound care was $46 \%$ (responder) and $26.5 \%$ (non-responders), while the probability of wound healing after 52 weeks of treatment was $82 \%$ (responder) and $64.9 \%$ (no responder). ${ }^{8}$

Research by Pietro et al., ${ }^{9}$ found that there was a relationship between CRP levels and the wound healing process. An increase in CRP levels indicates a severe inflammatory process (complications such as infection). In another study by Sang KC et al., one regarding factors related to the length of stay in diabetic foot patients, obtained a moderate correlation between the number of leukocytes with the degree of injury $(r=0.472)$ and moderate correlation also between CRP levels and degree of injury $(r=0.443)$ and both had a significant relationship $(p<0.05)$, and there was no significant relationship between the value of ESRs with the degree of injury $(p=0.088) .1$ In this study there was no statistically significant relationship but showed a moderate correlation between the relationship between leukocyte counts $(p=0.058 ; r=0.350)$ with changes in diabetic foot injury. This study did not follow previous studies conducted by Sang KC et al. regarding the relationship of leukocytes to wound area changes. In this study, there were not enough samples, and there were also many confounding factors.

A significant relationship was found between changes in CRP values with changes in diabetic foot injuries $(p=0.008 ; r=0.477)$ with moderate correlations. Likewise, the ESR was significantly correlated and moderately correlated $(p=0.034$; $r=0.388$ ) with changes in diabetic foot injury.
Many factors affect the condition of diabetic foot sores in patients, including the condition of the patient's vascular disorders, hyperglycemia, decreased immunity, smoking, hypoalbuminemia, and nutrition. Also, the factor of wound care carried out by the patient, and the patient's family indeed determines the wound healing process. ${ }^{10-13}$

\section{CONCLUSION}

There was a significant relationship between changes in ESR values, CRP values with changes in wound area with moderate correlation strength. There was no significant relationship between changes in the number of leukocytes with changes in the wound area. Further research related to other factors such as adequate blood sugar control and proper wound care needs to be done.

\section{CONFLICT OF INTEREST}

The author states the original work, and there is no conflict of interest in doing this research.

\section{ORCID ID OF AUTHORS}

Patrianef Darwis

https://orcid.org/0000-0002-4982-9034

Jimmy Candra Putra

https://orcid.org/0000-0003-1250-0785

Dedy Pratama

https://orcid.org/0000-0003-2043-3084

Aria Kekalih

https://orcid.org/0000-0001-7811-097X

\section{REFERENCES}

1. Choi SK, Kim CK, Jo DI, Lee MC, Kim JN, Choi HG, et al. Factors associated with a prolonged length of hospital stay in patients with diabetic foot: A single-center retrospective study. Arch Plast Surg. 2017;44(6):539-44.

2. Wild S, Roglic G. Global prevalence of diabetes: estimates for the year 2000 and projections for 2030. Diabetes Care. 2004;(27):1047-53.

3. Kementerian Kesehatan Republik Indonesia. Hasil utama riskesdas 2018. Jakarta: Badan Penelitian dan Pengembangan KEseh; 2018.

4. Jeffcoate W, Harding K. Diabetic foot ulcers. Lancet. 2003;361:1545-51.

5. Gerhard-Herman M, Gornik H, Barrett C, Barshes N, Corriere M, Drachman D. 2016 AHA/ACC guideline on the management of patients with lower extremity peripheral artery disease: executive summary: a report of the American College of Cardiology/American Heart Association Task Force on Clinical Practice Guidelines. Circulation. 2016.

6. Boulton A. The diabetic foot: grand overview, epidemiology and pathogenesis. Diabetes Metab Res Rev. 2008;24(Suppl. 1):3-6.

7. World Health Organization. Diabetes [Internet]. 2018 [cited 2019 Jul 22]. Available from: https://www.who.int/news-room/fact-sheets/detail/diabetes.

8. Coerper S, Beckert S, Küper MA, Jekov M, Königsrainer A. Fifty percent area reduction after 4 weeks of treatment is a reliable indicator for healing - analysis of a single-center cohort of 704 diabetic patients. J Diabetes Complications. 2009;23:49-53.

9. Pietro S, Dini V, Kirchhain A, Janowska A, Oranges T, Chiricozzi A. Sensors and biosensors for Creactive protein, temperature and $\mathrm{pH}$, and their applications for monitoring wound healing: A review. Molecular diversity preservation international. 2017;17:2-27.

10. Kayssi A, Rogers L, Neville R. General consideration of diabetic foot ulcers. In: Rutherford's vascular surgery and endovascular therapy. 9th ed. Philadelphia: Elsevier; 2019. p. 1950-62.

11. Murphy H. Inflammation. In: Rubin R, Strayer D, editors. Rubin's Pathology Clinicopathologic Foundations of Medicine. 6th ed. Philadelphia: Wolters Kluwer; 2012. p. 47-82. 
12. Pincus M, Abraham N. Interpreting laboratory results. In: McPherson R, Pincus M, editors. Henry's Clinical Diagnosis and Management by Laboratory Methods. 22nd ed. Philadelphia: Elsevier; 2011. p. 91-108.

13. Manna B, Morrison CA. Wound Debridement [Internet]. 2019 [cited 2019 Sep 1]. Available from: https://www.ncbi.nlm.nih.gov/books/NBK507882/ 